\title{
In patients with shortened dental arches do removable dental prostheses improve masticatory performance?
}

\author{
Abstracted from \\ Liang S, Zhang Q, Witter DJ, Wang Y, Creugers NH. \\ Effects of removable dental prostheses on masticatory performance of subjects with \\ shortened dental arches: A systematic review. J Dent 2015; 43: 1185-1194. doi: 10.1016/j.jdent.2015.05.008. \\ Epub 2015 Jun 8. Review. PubMed PMID: 26066662. \\ Address for correspondence: Department of Oral Function and Prosthetic Dentistry, \\ College of Dental Science, Radboud University Nijmegen Medical Centre, Philips van Leydenlaan 25, \\ 6525 EX Nijmegen, The Netherlands. E-mail. nico.creugers@radboudumc.nl
}

\section{Question: What are the effects of removable dental prostheses on masticatory performance in patients with a shortened dental arch?}

Data sources PubMed, Medline, Cochrane Library, Embase and Science Direct.

Study selection Studies published in English providing data on patients with shortened dental arch (SDA) and extreme SDA (ESDA) and masticatory performance with removable dental prostheses (RDP) were included. Data extraction and synthesis Two reviewers independently selected studies and abstracted data, with disagreements being resolved by discussion.

Results Eight studies were included; four reported on comminution studies, three on mixing ability and one included both. In patients with ESDA comminution or mixing ability was $28-39 \%$ lower compared to patients with a complete dentition. In two studies, comminution outcomes when chewing with an RDP ranged from $2 \%$ to $32 \%$ reduction, indicating better chewing function (smaller $\mathrm{X}_{50}$ ) compared to comminution without the RDP. One study reported $28-83 \%$ lower mixing ability when chewing at the RDP side than chewing at the dentulous side. Generally, more artificial teeth (or longer occlusal platform) in experimental RDPs resulted in better comminution and better mixing ability (significant in four out of five studies), indicating a 'dose-effect' relationship. Two of the eight studies reported on biting force with higher maximum occlusal force for biting with natural teeth than for biting with artificial teeth in a distal-extension RDP.

Conclusions Within the limitations of this review it can be concluded that subjects with (E)SDA had a reduced masticatory performance in the order of $30-40 \%$. Distal-extension RDPs compensated for this reduction only partially, namely in the order of 50\%. RDP effects on chewing frequency were not conclusive.

\section{Commentary}

For many patients replacement of missing teeth may be indicated to restore oral function, aesthetics and improve quality of life. Unfortunately, there is a limited amount of evidence and little consensus at present about how best to replace missing teeth in such patients. ${ }^{1}$ One option is utilisation of the Shortened Dental Arch (SDA) concept which aims to provide a functional dentition without the need for a removable partial denture (RPD). ${ }^{2}$ Multiple iterations of the SDA exist based on the number and distribution of remaining pairs of occluding teeth.
The aim of this systematic review was to summarise the impact of RPD provision on objective measures of masticatory function in SDA and extreme SDA (eSDA) patients. The review question, inclusion criteria and search strategy were clearly defined and a reasonable publication-date restriction was applied. A total of eight studies were identified and included. However, the sample sizes of the included studies were small, ranging from 8-30 patients.

Based on data reviewed from two studies, the effect of RPD provision on comminution appears to be inconclusive for SDA patients. One study reported an improvement in comminution, whilst the other a deterioration, post-RPD provision. One single study showed a 20\% improvement in comminution for eSDA patients provided with a RPD. Two further studies demonstrated an increase in comminution for eSDA patients provided with RPDs with a greater number of artificial teeth. A similar relationship was found for chewing function and the number of artificial teeth in two separate studies analysing SDA and eSDA.

This systematic review illustrates the paucity of high quality evidence to answer this research question. There was significant clinical and methodological heterogeneity amongst the small number of included studies. Unfortunately an assessment of the 'risk of bias' was not included. Only two studies analysed participants who did not act as their own controls and neither incorporated randomisation at the allocation stage. The authors accept that whilst the masticatory ability of SDA and eSDA patients may be lower than that of subjects with a complete dentition, the overall prevalence of chewing complaints is low in this population. ${ }^{3}$ Therefore in the first instance, patients with eSDAs who report chewing problems could be restored to a conventional SDA of ten occluding pairs using fixed prosthodontics. This approach has been demonstrated to be more cost-effective than RPDs whilst also achieving positive impacts on quality of life. ${ }^{4,5}$

Ciaran Moore and Gerry McKenna Centre for Public Health, Queens University Belfast, Northern Ireland

1. Abt E, Carr AB, Worthington HV. Interventions for replacing missing teeth: partially absent dentition. Cochrane Database Syst Rev 2012; (2): CD003814.

2. Käyser AF. Shortened dental arches and oral function. J Oral Rehabil 1981; 8: 457-462.

3. Sarita PT, Witter D], Kreulen CM, Van't Hof MA, Creugers NH. Chewing ability of subjects with shortened dental arches. Community Dent Oral Epidemiol 2003; 31: 328-334.

4. McKenna G, Allen F, Woods N, et al. Cost-effectiveness of tooth replacement strategies for partially dentate elderly: a randomized controlled clinical trial. Community Dent Oral Epidemiol 2014; 42: 366-374

5. McKenna G, Allen PF, O'Mahony D, Cronin M, DaMata C, Woods N. The impact of rehabilitation using removable partial dentures and functionally orientated treatment on oral health-related quality of life: a randomised controlled clinical trial. J Dent 2015; 43: 66-71.

Evidence-Based Dentistry (2016) 17, 114. doi:10.1038/sj.ebd.6401204 